\title{
View, play and pay? - The relationship between consumption of gaming video content and video game playing and buying
}

\author{
Maria Törhönen \\ Tampere University \\ maria.torhonen@tuni. \\ fi
}

\author{
Max Sjöblom \\ Tampere University \\ max.sjöblom@gmail. \\ com
}

\author{
Jukka Vahlo \\ Tampere University \\ jukka.vahlo@tuni.fi
}

\author{
Juho Hamari \\ Tampere University \\ juho.hamari@tuni.fi
}

\begin{abstract}
Consuming live-streamed or pre-recorded gaming video content through video sharing services such as YouTube and Twitch has become a significant aspect of the modern gaming and online media culture. Although it has been argued that watching such content may replace some first-hand gaming activities, it has also been argued that consuming gaming video content may further increase both gaming activities and purchases related to gaming. This study provides the first empirical evidence on how the consumption of three popular types of gaming video content (game reviews, let's plays and esports) affect the gameplay activity and game related purchasing behaviour and therefore extends our knowledge about digital consumer behaviour and its latest forms. Results indicate that all three forms of gaming video content have a positive association with time spent playing games. Moreover, gaming video consumption, especially the consumption of review video content, is positively associated with game purchasing behaviour.
\end{abstract}

\section{Introduction}

The development of online cultures and digital media formats have resulted in the convergence of different media formats and content. A prime example of such convergence is the emergence of gaming video content on digital video sharing platforms such as YouTube and Twitch [17, 32, 39], that merge the content of video games and the streaming/video content broadcasting phenomena.

This type of gaming video content is primarily focused on the topic of video games, and often highlights the gaming activities of private individuals (commonly referred to as Streamers, Broadcasters or YouTubers, depending on the format of video they distribute and the video sharing platforms they utilize). The popularity of gaming video content has increased in recent years, already reaching over 665 million people in 2017 [41]. This popularity is largely due to the emergence of dedicated video sharing platforms such as Twitch, Mixer and YouTube Gaming that are more specifically targeted to gaming video content and audiences.

The gaming video content may focus on video game content and gameplay, but can also revolve around topics related to the digital gaming culture at large (e.g., conventions and events related to games, esports tournaments etc.). The content of these videos often reflects the subjective experience of the content creator (game streamer or broadcaster) and therefore extends the original media content and its value for the consumer of these videos. The majority of game video content consumers $(73 \%)$ have been found to have a previous relationship with video game culture [28]. However, little is known about the appeal and the effect of gaming video content on the consumption of video games. Previous research on gaming video content consumption has primarily focused on the motivations to consume said content $[10,12,37]$, but more advanced behavioural research is not widely available yet.

The purpose of this research, therefore, is to examine how the consumption of three popular genres of gaming video content affects the actual gaming activity of a consumer and their game related purchasing behaviour. This research allows us to extend our knowledge about the digital consumer and the cross-media effects of digital video content. The data for this study was collected through an online survey $(\mathrm{N}=845)$ and the study analyses the effect of three genres of gaming video content: let's play, review, and esports, chosen for the analysis due to their popularity among consumers, their commercial potential and influence and their presence on the video sharing platforms [27, 31, 34]. The analytical 
model is based on previous understanding of gameplay behaviour $[3,9,45,49,50]$, game video content consumption [8,10,11, 12, 14, 37] and previous understanding of consumer behaviour [13, $21,29]$.

\section{Background}

Video game culture is a new media subculture which already rivals the film industry in revenue [24]. The development of this immersive subculture has been spearheaded by the emergence of new technology such as mobile technology and the internet, and has been characterized by the consistent convergence of computer technology, media products and human interaction [6, 28]. This has resulted in the emergence of new forms of video game content, such as gaming video content.

The emergence of YouTube in 2005 initiated a new trend in digital media and computer-mediated human interaction, that of social video content creation. The creators of social video content are primarily individuals, who produce and distribute (live or pre-recorded) video content through digital video sharing platforms such as YouTube and Twitch (among others) to social networks. The emergence of social video content creation has generated various niche trends and video content genres on popular video sharing platforms. Of these gaming video content begun to gain popularity soon after the emergence of YouTube [16].

Gaming video content has increased in popularity through the development of live streaming technology and dedicated game video sharing platforms such as Twitch, Mixer and YouTube Gaming. The emergence of electronic sports (esports) [42], (competitive video gaming), has also had a significant impact on generating global awareness of gaming video content, and has also led the rise of specific subgenres of video gaming content such as esports video content. Other popular subgenres that have emerged from gaming video content are let's play videos $[26,31]$ and review videos. Similarly to esports video content, these sub genres have had a significant impact on shaping gaming video content on video sharing platforms [31], and generating awareness of gaming video content. These popular subgenres were selected for inclusion in this study, as they all have different commercial potential and reflect different aspects of gaming video content.

Let's play videos emerged in 2006 have become a popular subgenre of gaming video content. Let's play videos are usually a compilation of gameplay video and commentary by the broadcaster, where the broadcaster may set out to explore, promote, critique or satirize a game or parts of a game. Although these videos highlight the video game itself, there is often a greater focus on the broadcaster as the narrator of the game and their interaction with the viewers. Let's play videos are often explorative in nature and rarely provide a structured overview of specific games, unlike review videos.

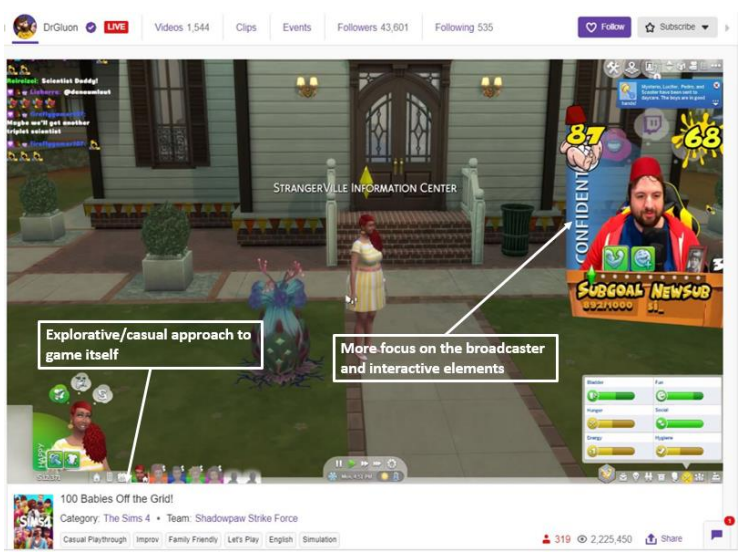

Image 1. Let's play video example

Game review videos usually consist of a subjective and relatively structured analysis/overview of specific game titles. Although the broadcaster functions as a narrator and the reviewer in these videos, game review videos are more specifically aimed at examining the video game itself and the focus is heavily on the game content. Game review videos are highly informative in nature, and provide a platform for the consumer to evaluate the video game as a product before purchase. This has also led to the development of direct commercial partnerships and promotions, where game developers/publishers may offer broadcasters the opportunity to review their game in exchange for a free copy of the game or even monetary compensation.

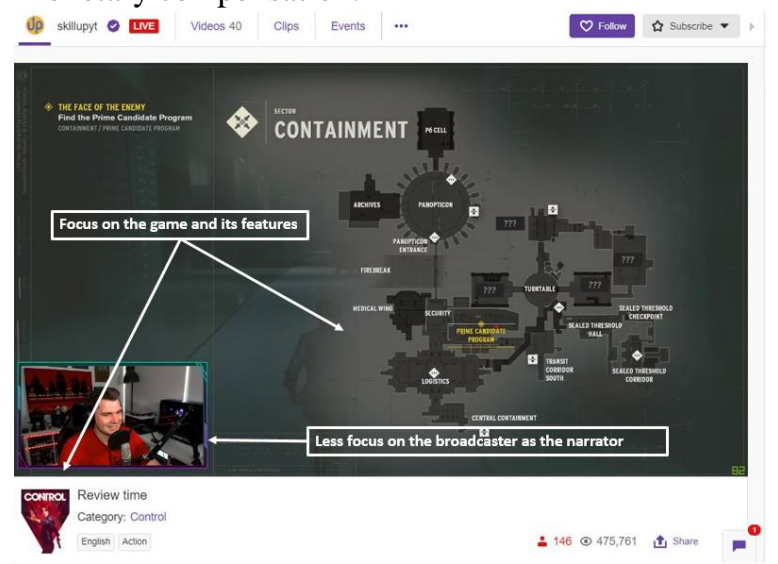

Image 2. Game review video example 
Esports (electronic sports) video content displays competitive video gaming, such as different esports events and tournaments or the training efforts/matches of esports players. Esports video content differs from let's play video content and review video content in its composition.

Esports video content usually consists of a separate commentator of the competition or the training efforts, the video game as the stage for the competition and the esports players. The commentator of esports video content acts similarly as the broadcaster/game streamer in let's play or review video content, but is rarely the focus of the video content itself and does not actually have control over the gameplay. The video game content acts as a stage for the competition, but the focus of the video is on the competitive gameplay, which is done by the esports players. These players are usually given a secondary visual element within the video content, which is used to construct a competitive setting, as seen in image 3 . The actions of the players are displayed on the digital game stage and they very rarely narrate or comment on the game during the competition.

Unlike review videos, the esports gaming video content does not directly promote the video game itself, but does display the game as a the stage for the competition, as mentioned. However, esports video content does often feature sponsored content, product placement, marketing and advertising efforts and therefore may have an indirect and visual commercial effect. As seen in image 3.

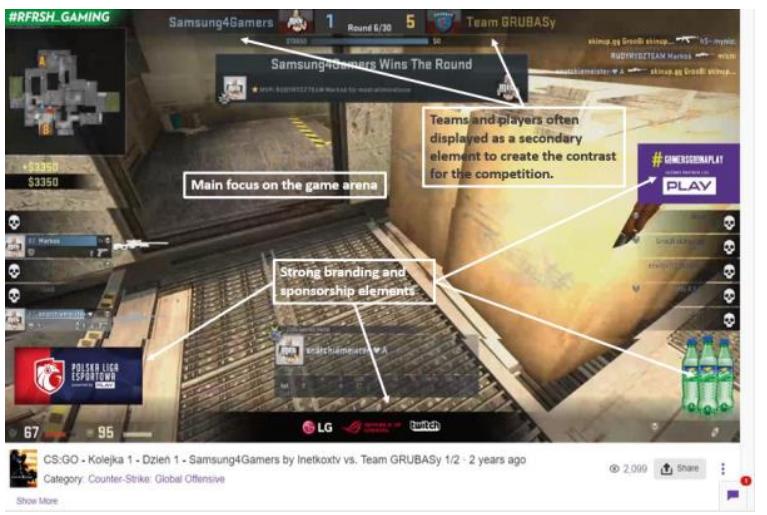

\section{Image 3. Esports video example}

The gaming video genre has become one of the most popular and profitable genres on YouTube [16], and the growing demand for this type of content has generated substantial commercial interest in game video content. Esports content alone was found to account for $11.6 \%$ of viewed hours on Twitch and $8.2 \%$ of viewed hours on YouTube Gaming [30].

\subsection{Previous research and consumer behaviour}

2.2.1 View, Play and Pay. With the rapid development of video game culture player behaviour, and especially game-related consumer behaviour have become a popular topic of research over the past decade, examining elements such as player-types and behaviours [3, 45], gaming preferences [15], and motivations to play [40, 48, 50].

Despite recent research efforts on the broader topic of social video content and consumer behaviour (especially in Asia) [43, 51], relatively little is still known about consumer behaviour related to gaming video content and video games. Research related to the consumption of gaming video content has primarily focused on the motivations to consume said content $[8,10,12,14,37,47]$ and the social behaviour related to gaming video content [12].

Previous research on consumer behaviour [13, 21, 29] has modelled the decision making process of a consumer numerous times, in order to define the different stages of a decision-making process [29]. Although many of these efforts have been based on the assumption that the consumer makes decisions that are founded on rational analytical processing, they have been successful in describing and analysing different purchasing behaviour as well as different elements attributing to such behaviour.

The majority of research on purchasing and consumer behaviour describes the stage leading up to a purchase, as a decision-making or informationseeking phase [21, 29] in which the consumer evaluates internal needs and wants and may be particularly susceptible to external stimuli (social, cultural, commercial). Research on gaming video content has found that a strong motivator in gaming video consumption is that of information-seeking, as well as the social interaction [12, 37]. Accordingly, we anticipate that this form of content will be a particularly strong influencer in this pre-purchase phase. Research related to other types of social video content and the content creators have also revealed that social video content creators have a strong influence on their audiences and their consumer behaviour [7, 18, 23].

Based on the discussion above, we hypothesize the following:

H1: The consumption of review video content has a positive association with game related purchasing behaviour. 
H2: The consumption of esports video content Review video content has a positive association with game related purchasing behaviour.

H3: The consumption of let's play content has a positive association on game related purchasing behaviour.

As with the pre-purchase stages, research on consumer decision-making processes have also described post-purchase behaviour [21]. This occurs after the purchase of a product (such as a video game) and consists of the use and re-evaluation of the product, which in the context of this research would describe video gameplay activities [46]. Previous research on social video content consumption has also found that social interaction and learning are significant motivators for video content consumption $[8,12,37]$, making gaming video content a potential source of new strategies and community building, which may be highlighted in further gameplay due to consumption of gaming video content.

Based on the discussion above, we hypothesize the following:

H4: The consumption of review video content has a positive association with gameplay activity

H5: The consumption of let's play video content has a positive association with gameplay activity

H6: Esports video content has a positive association with gameplay activity.

\section{Methods and Data}

\subsection{Sampling}

A total of 845 survey respondents (ages 18-55, $49.2 \%$ male, $50.8 \%$ female) were obtained from Finland to construct a representative sample of the core adult gaming population of the country. Please see Appendix A for a more detailed breakdown of the demographic data. The Finnish gaming industry is one of the fastest growing in the global gaming market with an average growth rate of $45 \%$ [22], resulting in a rapidly developing gaming culture. Over $70 \%$ of the population reported playing digital games in 2018, with $30.6 \%$ of men reported to watching gaming related video content [20].

The survey was conducted in cooperation with a market research company (M3 Finland), by using a web-based tool. The market research company eliminated any responses deemed unsatisfactory based on response time, before data was accessed by the research team. Participants were also asked to report their level of interest in digital games and gaming on a 5 -point Likert scale $(1=$ not at all interested, $5=$ very interested). In order to ensure valid responses participants were disqualified from completing the survey if they stated that they were not at all interested in digital games or gaming.

Survey respondents were instructed to estimate their game video consumption by asking how often they watch different gaming video sub-genres (esports, let's play or review) on a pre-determined 6point scale $(1=$ not at all, $6=$ multiple times a week). The respondents were also asked to estimate how much time they spend playing video games per week on a pre-determined scale (hours and minutes), and how much money they spent on gaming and game related purchases per month, on a pre-determined 8point scale $(1=$ not at all, $8=$ over 200 euros $)$. Different scales were used to measure different variables in order to highlight the activity in question. For example, the scale to measure money spent on game related purchases was developed to best reflect game related purchasing and the 8-point scale was based on different reports about game related purchasing behaviour.

\subsection{Structural Model \& Analysis}

In order to assess the effect of watching gaming video content on gameplay activity and game related purchasing, we constructed a structural model (Figure $1)$.

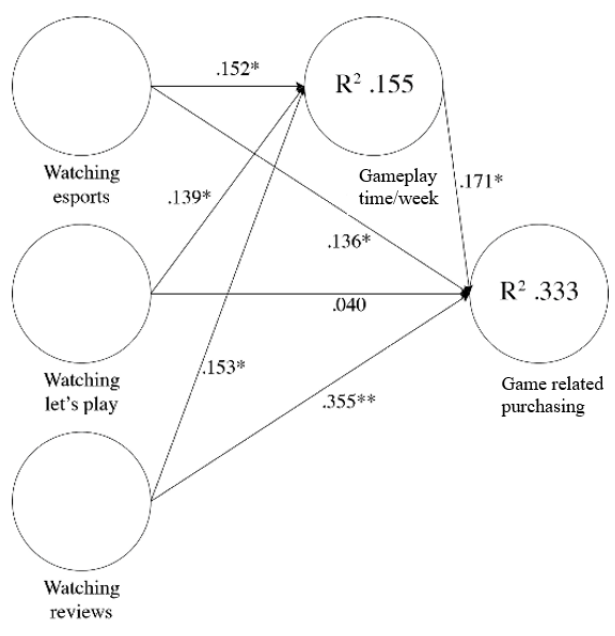

Figure 2. Structural model with results ( ${ }^{*} p$ $<.05,{ }^{\star \star} \mathrm{p}<.001$ ) 
As previously detailed, the three independent variables examining estimated time spent on consuming the three sub-genres of gaming video content, were all measured on a 6-point frequency scale. The total time spent playing games was an estimated amount of hours and minutes per week, provided on a pre-determined list of hours and minutes. The dependent variable measuring gamerelated purchases was constructed using the estimated amount of money (euros) spent on gaming and game related purchases per month, which was provided on a pre-determined 8-point scale. For this study, the analysis was conducted using the structural equation modelling software SmartPLS 3.2.6 [35]. Pairwise deletion of missing data was used in order to maintain a high level of reliability within the data.

\section{Results}

The model accounted for $15.5 \%$ of the variance for the time spent playing games and $33.3 \%$ of the variance for spending money on games. Watching esports $\left(\beta=.152^{*}\right)$, let's play $\left(\beta=.139^{*}\right)$ and review video content $\left(\beta=.153^{*}\right)$ all had a statistically significant positive association with the amount of time spent playing games. For spending money on games, esports $\left(\beta=.136^{*}\right)$ and review video content $\left(\beta=.355^{* *}\right)$ both showed a statistically significant positive association, while let's play videos did not have a statistically significant association. Furthermore, when examining the link between time spent playing games and the amount spent on game related purchases $(\beta=.171 *)$, a positive and statistically significant association was found. All results are displayed below in Table 1.

\begin{tabular}{lllllll}
\hline & \multicolumn{2}{c}{ Gameplay time/week } & \multicolumn{3}{c}{ Game related purchasing } \\
\hline R2 & \multicolumn{5}{c}{.155} \\
\cline { 2 - 7 } & $\beta$ & $\mathrm{CI}$ & $\mathrm{p}$ & $\beta$ & $\mathrm{CI}$ & $\mathrm{p}$ \\
\hline $\begin{array}{l}\text { Esports } \\
\text { Let's }\end{array}$ & $.152^{*}$ & $.033-.264$ & .009 & $.136^{*}$ & $.019-.260$ & .027 \\
play & $.139 *$ & $.016-.257$ & .023 & .040 & $-.050-.135$ & .399 \\
$\begin{array}{l}\text { Review } \\
\begin{array}{l}\text { Playing } \\
\text { games }\end{array}\end{array}$ & $.153^{*} .040-.272$ & .009 & $.355^{* *}$ & $.235-.470$ & .000 \\
\hline
\end{tabular}

\footnotetext{
Table 1. Results of PLS-SEM analysis ( ${ }^{*} p$ $\left.<.05,{ }^{\star \star} p<.001\right)$
}

\section{Discussion}

The results of this research indicate that both esports and review videos have a statistically significant, positive association with game related purchases (H1 and $\mathrm{H} 2$ not rejected). However, there was no association between let's play video content and game-related purchasing behaviour, meaning hypothesis $\mathrm{H} 3$ was rejected. All three types of game video content were also found to have a significant positive association with playing games ( $\mathrm{H} 3, \mathrm{H} 4$ and H5 not rejected).

\subsection{Esports and consumer behaviour}

The structures of electronic sports and sporting events have adopted several elements from traditional spectator sports [42] and commercial elements are also similar (e.g. visual sponsorship placement on game arena, sponsored teams etc.) [44]. It should be noted that, in addition to traditional sponsorship placements, esports features a commercial product at the centre of esports itself (the video game being played) and esports events and tournaments are also often produced with commercial third-party representatives (such as the game developers or event organisers).

The effect of commercial messaging in esports may be heightened due to the additional levels of community/social stimuli provided by the gaming video content [39], and through interaction with the possible narrator/broadcaster and community through chat features on platforms such as Twitch. Through the consumption of esports video content the consumer may therefore be subjected to various sponsored and branded messages, which may affect the consumer in various stages of the decision making process. Visual sponsorship, advertising and product placement have been found to have a significant effect on consumer behaviour and purchasing in traditional spectator sports in the past $[4,33]$. Also, attachment to a specific esports teams may encourage the consumer to support their team through the purchase of sponsored items, similarly as in traditional sports the strategic team sponsorship and product endorsement have been found to affect the consumer behaviour of sports fans $[4,5]$.

Esports video content also portrays the best players of specific games displaying their skills and often their strategies and tactics. As such, it may provide further incentive to test these strategies through gameplay, and also lead to purchases of ingame items that may improve gameplay skills or mimic gameplay strategies [37, 38]. 
In practice, the development of games directed towards esports viewing could further emphasise the commercial potential of product placement and branding within the games themselves. Although this is already done to some extent [42], there is still potential to expand this further by allowing branding or personalisation of e.g. specific in-game content, characters or game arenas. This could emphasise the commercial effect for the community and generate a closer association between fans and specific games and gaming products.

Although this research reveals a significant effect between esports video content consumption and consumer behaviour (purchasing and gameplay activity), more research could be directed to specific purchasing behaviour such as in-game content purchases (skins, loot etc.) or the purchase of gaming-related merchandise. In this way, the effect and potential of this type of gaming video content could be better understood.

\subsection{Review gaming videos and consumer behaviour}

As review videos are specifically generated for evaluating games and their content, they provide an ideal source for consumers to gather product information. Most review video content is generated by individual content creators, and direct commercial incentive or collaboration with a commercial entity should be stated depending on the local law or platform regulations and ad policies [2]. This may make review video content feel more transparent, relatable and authentic, as already shown in research related to "influencers" $[1,7]$.

The relatability and authenticity of video content creators as private individuals has led to the emergence of the "influencer" culture, where content creators are considered to have an influence on their audiences and their consumer behaviour $[1,7,25]$. According to recent research, a message delivered by a social media "influencer" can be much more effective than a traditional marketing message, as it is delivered by someone relatable and close to the audience $[1,19,36]$. This influencer-effect within review video content may affect consumer behaviour in various stages of the purchase decision making process $[13,21,29]$. It may generate a need for a specific video game product, but may also provide the necessary information about the product that leads to a purchase.

The finding related to review video content and purchasing behaviour emphasises the power of video content creators as commercial influencers. Although the content creators maintain the liberty to describe and review the video game content in a manner they prefer (also display negative responses to products), the review video content seems to have the ability to act as a personalised commercial message even without direct commercial agenda.

As sources of information, and a subjective experience of a video game, review videos also provide a way to experience the video game itself. Although it could be argued that this exposure to the game content may dilute the gaming experience itself, our findings indicate that review videos actually encourage gameplay. Naturally, viewers of review videos may be looking for encouraging comments about a game and be motivated to test the game themselves, but review videos also only provide a subjective experience of the game and therefore may motivate and encourage further exploration of the game itself.

In future research a more controlled study could be conducted on the consumption behaviour of a specific game title, in order to analyse the effects of watching review videos on the gameplay experience and behaviour itself.

\subsection{Let's play gaming video content and consumer behaviour}

The findings of this research reveal that there was no significant relationship between let's play video consumption and game-related purchases, although watching let's play content does have an effect on the gameplay activity. This finding reveals that there are some differences between the effects of different gaming video sub-genres on consumption behaviour.

The let's play video content is similar to review video content in nature, as it often explores a specific video game. Unlike review videos, however, the focus is not purely on the video game itself, but more on the content creator and the interaction with the audience. The interactive nature of let's play content has generated popularity for various individual content creators [26], and often develops into an active community. Although the social stimuli is evident in this type of content, the lack of clear and purposeful commercial messaging may not generate the same effect that can be seen for example in esports fan communities, but the content thrives on the entertaining combination of gameplay and interaction. As the focus of this type of content is on entertainment, consumers may not be as inclined to consume let's play content for informative purposes either.

Interestingly, this analysis discovered that consumption of let's play video content was associated with the time spent playing games. 
Similarly, to review video content, let's play video content provides an explorative experience of the video game being played. However, the gameplay is rarely structured to provide an overview of the entire game, and often aims to provide an entertaining experience of the video game, which could motivate viewers to play the game themselves.

As mentioned above, let's play content differs from the other types of game video content examined in this research, as it does not have the same natural commercial aspect as esports video content or review video content. However, let's play content does have a strong social aspect, in the form of community involvement and the conversational nature of the content, which could be considered to allow for social stimuli similar to other content examined in this research. Based on this finding, there is a need for more structured analysis of different game video genres, and evaluation of their commercial potential and association. In the future, it would be interesting to also further examine the causality between the variables, to examine the relationship of playing games and consuming gaming video content and making game related purchases.

\section{Conclusion}

This study investigated the effects of gaming video content consumption (specifically esports video content, review video content and let's play video content) on both game-related purchasing behaviour and gameplay. The model used in this study was based on previous research and understanding of gaming video content consumption, consumer behaviour and player behaviour [13, 21, 29].

The data for this study $(\mathrm{N}=845)$ was gathered through an online survey and the respondents were primarily gathered from Finland. The qualified respondents all had previous experience with games and gaming. The results of the study indicated that the consumers of esports and review game video content are likely to make game related purchases, whereas the consumption of let's play content does not have a similar effect on consumer behaviour. We conclude that esports and review game video content differ from let's play game video content in their commercial nature, as esports and review game video content have a more direct commercial aspect through e.g. sponsorship (in esports content) and product representation (in review video content). These videos are also more informative in nature and present a strong social stimulus through community involvement, which may affect consumer behaviour [21]. Let's play videos often focus more on the video content creator and their interaction with the audience, and the commercial aspect is not as evident.

The results of the study also indicated that the consumption of all examined types of game video content were associated with playing games. We conclude that this finding may reveal that players gain new perspectives from game video content that they then may want to either test or try through their own gameplay. We also conclude that the game video content provides new social dimensions to game content, which has been known to motivate gameplay $[3,48,49,50]$.

\section{Limitations}

It should be noted that this study aims to examine the direct relationship between the consumption of gaming video content, gameplay activities and gaming related purchases. The analysis of this research is therefore based on the previous understanding of consumer behaviour, which often assumes consumer behaviour as a rational and a linear process. However, we understand that consumer behaviour may not always be based on rational thinking and many of the stages of the decision making process may happen simultaneously. Therefore, there may be indirect effects between the variables examined in this study and this analysis could be developed to examine the causality of these relationships in the future.

As this study is focused on understanding the consumption of game video content and its effects on gameplay and game-related purchasing behaviour, a survey was considered a suitable method for data collection. A survey provides an effective way to collect personal perceptions and views of reality, but also has certain limitations. The main limitation of an online survey is that the self-reported responses cannot be monitored in any way as the responses are made in an unsupervised and possibly distracted environment. This limitation was taken into consideration by using a market research company to collect the data and make necessary steps to ensure the best validity of data as possible.

Nonetheless, in the future, a wider array of research methods such as qualitative focus groups or interviews could provide more nuanced insight into this activity. Ethnographic observation could also ensure a more in-depth insight into these activities. However, it should be noted that the intensity of this behaviour combined with its private nature, would make the use of these research methods difficult and provide further limitation to the study of this topic. Another limiting factor of this study is the geographic 
targeting of the survey, as it focuses on consumers in Finland. This research was only aimed at players, who deem themselves as somewhat active in their gaming activities. It should be noted that the survey did not examine those consumers with no previous gaming background or lower levels of activity, and this could be and interesting topic for future research.

\section{References}

[1] Abidin, C., and M. Ots, "The Influencer's dilemma: The shaping of new brand professions between credibility and commerce", Proceedings of The Association for Education in Journalism and Mass Communication (AEJMC) conference, San Francisco 2015, pp. 1-12.

[2] Alvarez, E., "YouTube stars are blurring the lines between content and ads", Engadget, 2017. https://www.engadget.com/2017/07/25/youtube-

influencers-sponsored-videos/

[3] Bartle, R. "Hearts, Clubs, Diamonds, Spades: Players who suit MUDs", Journal of MUD research, 1996.

[4] Biscaia, R., A. Correia, A.F. Rosado, S.D. Ross, and J. Maroco, "Sport Sponsorship: The Relationship between Team Loyalty, Sponsorship Awareness, Attitude Toward the Sponsor, and Purchase Intentions", Journal of Sport Management 27(3), 2013. pp. 288-302

[5] Crawford, G., Consuming sport: Fans, sport and culture, 2004.

[6] De Freitas, S., and M. Griffiths, "The convergence of gaming practices with other media forms: What potential for learning? A review of the literature", Learning, Media and Technology 33(1), 2008. pp. 10-20

[7] Gräve, J.-F., and A. Greff, "Good KPI, Good Influencer?: Evaluating Success Metrics for Social Media Influencers", Proceedings of the 9th International Conference on Social Media and Society (SMSociety '18), ACM, New York, NY 2018, pp. 291-295.

[8] Gros, D., B. Wanner, A. Hackenholt, P. Zawadzki, and K. Knautz, "World of streaming. Motivation and gratification on twitch", Proceedings of the International Conference on Social Computing and Social Media, Springer, 2017, pp. 44-57.

[9] Hamari, J., and J. Tuunanen, "Player Types : A Metasynthesis", Selected Articles from the DiGRA Nording 2012 Conference: Local and Global-Games in Culture and Society, 2014, pp. 29-53

[10] Hamilton, W.A., O. Garretson, and A. Kerne, "Streaming on twitch: fostering participatory communities of play within live mixed media", Proceedings of the SIGCHI Conference on Human Factors in Computing Systems, 2014, pp. 1315-1324.
[11] Hamilton, W.A., O. Garretson, and A. Kerne, "Streaming on twitch", Proceedings of the 32nd annual ACM conference on Human factors in computing systems CHI '14, 2014, pp. 1315-1324.

[12] Hilvert-Bruce, Z., J.T. Neill, M. Sjöblom, and J. Hamari, "Social motivations of live-streaming viewer engagement on Twitch", Computers in Human Behavior 84 (7), 2018. pp. 58-67.

[13] Howard, J.A., and J.N. Seth, A Theory of Buyer Behavior, 1969.

[14] Hu, M., M. Zhang, and Y. Wang, "Why do audiences choose to keep watching on live video streaming platforms? An explanation of dual identification framework", Computers in Human Behavior 75, 2017, pp. 594-606.

[15] Jansz, J., and M. Tanis, "Appeal of Playing Online First Person Shooter Games", CyberPsychology \& Behavior 10(1), 2007. pp. 133-136.

[16] Johnsmeyer, B., J. Getomer, and M. Okimoto, "Gamers on YouTube: Evolving Video Consumption", Think with Google, 2013. https://www.thinkwithgoogle.com/consumerinsights/youtube-marketing-to-gamers/

[17] Johnson, M.R., and J. Woodcock, "'It's like the gold rush': the lives and careers of professional video game streamers on Twitch.tv", Information Communication and Society, 2017. pp. 336-351.

[18] Johnstone, L., and C. Lindh, "The sustainability-age dilemma: A theory of (un)planned behaviour via influencers", Journal of Consumer Behaviour, 2018.pp.127-139.

[19] Khamis, S., L. Ang, and R. Welling, "Self-branding, 'micro-celebrity' and the rise of Social Media Influencers", Celebrity Studies 8(2), 2017, pp. 191-208.

[20] Kinnunen, J., P. Lilja, and F. Mäyrä, Pelaajabarometri 2018: Monimuotoistuva mobiilipelaaminen, 2018. pp.4144.

[21] Kollat, D.T., J.F. Engel, and R.D. Blackwell, "Current Problems in Consumer Behavior Research", Journal of Marketing Research 7(3), 1970. pp. 327-332

[22] Kostner, P., "Finland, still a gaming superpower in 2018", Helsinki 2019. https://www.helsinkitimes.fi/207-themes/player-one/16289finland-still-a-gaming-superpower-in-2018.html

[23] Langner, S., N. Hennigs, and K.P. Wiedmann, "Social persuasion: Targeting social identities through social influencers", Journal of Consumer Marketing 30(1), 2013.pp. 31-49. 
[24] Lufkin, B., "How video games turn teenagers into millionaires", $\quad$ BBC, 2018. http://www.bbc.com/capital/story/20180323-how-videogames-make-some-teens-millionaires

[25] Marwick, A.E., "You May Know Me from YouTube: (Micro-)Celebrity in Social Media", In in A Companion to Celebrity. 2015, pp. 100-127.

[26] McConnell, F., "Let's Play - the YouTube phenomenon that's bigger than One Direction", The Guardian, 2014. https://www.theguardian.com/technology/2014/jan/02/letsplay-youtube-pewdiepie-one-direction

[27] Mediakix, "THE DIFFERENT TYPES OF YOUTUBE GAMING VIDEOS”, Mediakix, 2015. https://mediakix.com/blog/youtube-gaming-videos-thedifferent-types/

[28] Newzoo, "The Consumer as Producer: How Games \& Video Converge to Drive Growth", Newzoo, 2015. https://newzoo.com/insights/articles/the-consumer-asproducer-how-games-video-converge-to-drive-growthwhitepaper/

[29] Nicosia, F.M., "Perceived Risk, Information Processing, and Consumer Behavior", Journal of Business 42(2), 1969. pp. 162-166

[30] Pannekeet, J., "Five Key Insights into Twitch and YouTube Gaming and the 2.4Bn Viewing Hours They Generated in Q1 2018", Newzoo, 2018. https://newzoo.com/insights/articles/five-key-insights-intotwitch-and-youtube-gaming/

[31] Park, G., 'Once 'a new art form,' Let's Play videos are facing a glut", The Washington Post, 2019. https://www.washingtonpost.com/arts-

entertainment/2019/05/03/once-new-art-form-lets-play-

videos-are-facing-

glut/?noredirect=on\&utm_term=.6ea8e $253 \mathrm{fdf} 7$

[32] Pellicone, A., "Performing Play: Cultural Production on Twitch.tv", Proceedings of the 2016 CHI Conference on Human Factors in Computing Systems - CHI '16, 2016, pp. 244-248.

[33] Pope, N.K., and K.E. Voges, "The Impact of Sponsorship Activities, Corporate Image, and Prior Use on Consumer Purchase Intention", Sport Marketing Quarterly 9(2), 2000. pp. 96-102

[34] Popper, B., "Field of streams: how Twitch made video games a spectator sport", The Verge, 2013. https://www.theverge.com/2013/9/30/4719766/twitchraises-20-million-esports-market-booming

[35] Ringle, C.M., S. Wende, and J.-M. Becker, "Ringle, Christian M., Wende, Sven, \& Becker, Jan-Michael.
(2015). SmartPLS 3. Bönningstedt: SmartPLS. Retrieved from http:/www.smartpls.com", Retrieved from, 2015, 2015. www.smartpls.com

[36] Senft, T.M., "Microcelebrity and the Branded Self", In A Companion to New Media Dynamics. 2013, pp. 346354.

[37] Sjöblom, M., and J. Hamari, "Why do people watch others play video games? An empirical study on the motivations of Twitch users", Computers in Human Behavior 75, 2017, pp. 985-996.

[38] Sjöblom, M., M. Törhönen, J. Hamari, and J. Macey, "Content structure is king: An empirical study on gratifications, game genres and content type on Twitch", Computers in Human Behavior 73, 2017. pp. 161-171.

[39] Sjöblom, M., M. Törhönen, J. Hamari, and J. Macey, "The ingredients of Twitch streaming: Affordances of game streams", Computers in Human Behavior 92, 2019. pp. 20-28.

[40] Souza, L.L.F. de, and A.A.F. de Freitas, "Consumer behavior of electronic games' players: a study on the intentions to play and to pay", Revista de Administração 52 (4), 2017. pp. 419-430.

[41] Taylor, H., "Gaming video content has an audience of 665 million”, Gamesindustry.biz, 2017. https://www.gamesindustry.biz/articles/2017-10-20superdata-report-finds-gaming-video-content-has-anaudience-of-665-million

[42] Taylor, T.L., Raising the Stakes: E-Sports and the Professionalization of Computer Gaming, MIT Press, 2012.

[43] Tong, J., "A Study on the Effect of Web Live Broadcast on Consumers' Willingness to Purchase", Open Journal of Business and Management 5(2), 2017. pp. 280289.

[44] Turtiainen, R., U. Friman, and M. Ruotsalainen, "NNot Only for a Celebration of Competitive Overwatch but Also for National Pride': Sportificating the Overwatch World Cup 2016", Games and Culture, 2018, pp. 1-21.

[45] Vahlo, J., J.K. Kaakinen, S.K. Holm, and A. Koponen, "Digital Game Dynamics Preferences and Player Types", Journal of Computer-Mediated Communication 22 (4), 2017. pp. 88-103.

[46] Vahlo, J., J. Smed, and A. Koponen, "Validating gameplay activity inventory (GAIN) for modeling player profiles", User Modeling and User-Adapted Interaction 28 (4-5), 2018. pp. 425-453.

[47] Wulf, T., F.M. Schneider, and S. Beckert, "Watching Players: An Exploration of Media Enjoyment on Twitch", Games and Culture, 2018, pp. 1-19. 
[48] Yee, N., "Facets: 5 motivation factors for why people

play MMORPGs",

Http://Www.Nickyee.Com/Facets/Home.Html, 2002.

[49] Yee, N., "Motivations for Play in Online Games", CyberPsychology \& Behavior 9(6), 2006. pp.772-775.

[50] Yee, N., "Motivations of Play in MMORPGs - Results from a Factor Analytic Approach", http://nickyee.com/daedalus/archives/pdf/3-2.pdf

[51] Zhang, M., F. Qin, G.A. Wang, and C. Luo, "The impact of live video streaming on online purchase intention”, Service Industries Journal, 2019. pp.1-27.

\section{APPENDIX A}

\begin{tabular}{|c|c|c|c|}
\hline$N$ & $\begin{array}{r}\text { Sample } \\
845\end{array}$ & $\begin{array}{r}\text { Male subsample } \\
416(49.2 \%)\end{array}$ & $\begin{array}{r}\text { Female } \\
\text { subsample } \\
429(50.8 \%)\end{array}$ \\
\hline$\overline{\text { Age }}$ & 34.6 & 36.9 & 32.3 \\
\hline Weekly play hours & 11.7 & 13.0 & 10.4 \\
\hline $\begin{array}{l}\text { Monthly } \\
\text { money spent }(€)\end{array}$ & 8.7 & 9.3 & 8.1 \\
\hline $\begin{array}{l}\text { Let's play } \\
\text { consumption }(\%)\end{array}$ & $45.5 \%$ & & \\
\hline $\begin{array}{l}\text { Game review } \\
\text { consumption }(\%)\end{array}$ & $53.4 \%$ & & \\
\hline $\begin{array}{l}\text { Esports consumption } \\
(\%)\end{array}$ & $36.2 \%$ & & \\
\hline $\begin{array}{l}\text { Esports video } \\
\text { viewing frequency }\end{array}$ & 1.7 & 1.9 & 1.5 \\
\hline $\begin{array}{l}\text { Let's play video } \\
\text { viewing frequency }\end{array}$ & 2.0 & 2.2 & 1.8 \\
\hline $\begin{array}{l}\text { Game review video } \\
\text { viewing frequency }\end{array}$ & 2.1 & 2.4 & 1.8 \\
\hline $\begin{array}{l}\text { Total game video } \\
\text { viewing frequency }\end{array}$ & 2.0 & 2.2 & 1.7 \\
\hline
\end{tabular}

${ }^{a}$ Values are reported as mean sums.

${ }^{b}$ Percentage of respondents who reported to consume content

${ }^{c}$ Frequency measured on a scale of 1-6 $(1=$ not at all, $6=$ multiple times a

week). 